\title{
How do we present messy syndromic surveillance data to public health's partners?
}

\section{David Atrubin*1, Rosa Ergas ${ }^{2}$ and Aaron Kite-Powell ${ }^{3}$}

'Florida Department of Health, Tampa, FL, USA; ${ }^{2}$ Massachusetts Department of Public Health, Boston, MA, USA; ${ }^{3}$ Centers for Disease Control and Prevention, Atlanta, GA, USA

\section{Objective}

To discuss data disclaimers and caveats that are fundamental to sharing syndromic surveillance (SyS) data

\section{Introduction}

With increasing awareness of SyS systems, there has been a concurrent increase in demand for data from these systems - both from researchers and from the media. The opioid epidemic occurring in the United States has forced the SyS community to determine the best way to present these data in a way that makes sense while acknowledging the incompleteness and variability in how the data are collected at the hospital level and queried at the user level. While significant time and effort are spent discussing optimal queries, responsible presentation of the data - including data disclaimers - is rarely discussed within the SyS community.

\section{Keywords}

data disclaimers; data sharing; variability within the data; syndromic surveillance; emergency department data

\section{*David Atrubin}

E-mail: david.atrubin@flhealth.gov 